\title{
Better Bootstrapping in Fully Homomorphic Encryption
}

\author{
Craig Gentry ${ }^{1}$, Shai Halevi ${ }^{1}$, and Nigel P. Smart ${ }^{2}$ \\ 1 IBM T.J. Watson Research Center \\ ${ }^{2}$ Dept. Computer Science, University of Bristol
}

\begin{abstract}
Gentry's bootstrapping technique is currently the only known method of obtaining a "pure" fully homomorphic encryption (FHE) schemes, and it may offers performance advantages even in cases that do not require pure FHE (e.g., when using the noise-control technique of Brakerski-Gentry-Vaikuntanathan).

The main bottleneck in bootstrapping is the need to evaluate homomorphically the reduction of one integer modulo another. This is typically done by emulating a binary modular reduction circuit, using bit operations on binary representation of integers. We present a simpler approach that bypasses the homomorphic modularreduction bottleneck to some extent, by working with a modulus very close to a power of two. Our method is easier to describe and implement than the generic binary circuit approach, and we expect it to be faster in practice (although we did not implement it yet). In some cases it also allows us to store the encryption of the secret key as a single ciphertext, thus reducing the size of the public key.

We also show how to combine our new method with the SIMD homomorphic computation techniques of Smart-Vercauteren and Gentry-Halevi-Smart, to get a bootstrapping method that works in time quasi-linear in the security parameter. This last part requires extending the techniques from prior work to handle arithmetic not only over fields, but also over some rings. (Specifically, our method uses arithmetic modulo a power of two, rather than over characteristic-two fields.)
\end{abstract}

\section{Introduction}

Fully Homomorphic Encryption (FHE) [127] is a powerful technique to enable a party to compute an arbitrary function on a set of encrypted inputs; and hence obtain the encryption of the function's output. Starting from Gentry's breakthrough result [6]7, all known FHE schemes are constructed from Somewhat Homomorphic Encryption (SWHE) schemes, that can only evaluate functions of bounded complexity. The ciphertexts in these SWHE schemes include some "noise" to ensure security, and this noise grows when applying homomorphic operations until it becomes so large that it overwhelms the decryption algorithm and causes decryption errors. To overcome the growth of noise, Gentry used a bootstrapping transformation, where the decryption procedure is run homomorphically on a given ciphertext, using an encryption of the secret key that can be found in the public key 11 resulting in a new ciphertext that encrypts the same message but has potentially smaller noise.

Over the last two years there has been a considerable amount of work on developing new constructions and optimizations [5|13|9|3|14 2|8|1|11], but all of these constructions

\footnotetext{
${ }^{1}$ This transformation relies on the underlying SWHE being circularly secure.
} 
still have noise that keeps growing and must be reduced before it overwhelms the decryption procedure. The techniques of Brakerski et al. [1] yield SWHE schemes where the noise grows slower, only linearly with the depth of the circuit being evaluated, but for any fixed public key one can still only evaluate circuits of fixed depth. The only known way to get "pure" FHE that can evaluate arbitrary functions with a fixed public key is by using bootstrapping. Also, bootstrapping can be used in conjunction with the techniques from [1] to get better parameters (and hence faster homomorphic evaluation), as described in [1|11].

In nearly all SWHE schemes in the literature that support bootstrapping, decryption is computed by evaluating some ciphertext-dependent linear operation on the secret key, then reducing the result modulo a public odd modulus $q$ into the range $(-q / 2, q / 2]$, and then taking the least significant bit of the result. Namely, denoting reduction modulo $q$ by $[\cdot]_{q}$, we decrypt a ciphertext $c$ by computing $a=\left[\left[L_{c}(s)\right]_{q}\right]_{2}$ where $L_{c}$ is a linear function and $s$ is the secret key. Given an encryption of the secret key $s$, computing an encryption of $L_{c}(s)$ is straightforward, and the bulk of the work in homomorphic decryption is devoted to reducing the result modulo $q$. This is usually done by computing encryptions of the bits in the binary representation of $L_{c}(s)$ and then emulating the binary circuit that reduces modulo $q$.

The starting point of this work is the observation that when $q$ is very close to a power of two, the decryption formula takes a particularly simple form. Specifically, we can compute the linear function $L_{c}(s)$ modulo a power of two, and then XOR the top and bottom bits of the result. We then explain how to implement this simple decryption formula homomorphically, and also how the techniques of Gentry et al. from [11] can be used to compute this homomorphic decryption with only polylogarithmic overhead.

We note that applying the techniques from [11] to bootstrapping is not quite straightforward, because the input and output are not presented in the correct form for these techniques. (This holds both for the standard approach of emulating binary mod- $q$ circuit and for our new approach.) Also, for our case we need to extend the results from [11] slightly, since we are computing a function over a ring (modulo a power of two) and not over a field.

We point out that in all work prior to [11], bootstrapping required adding to the public key many ciphertexts, encrypting the individual bits (or coefficients) of the secret key. This resulted in very large public keys, of size at least $\lambda^{2} \cdot \operatorname{polylog}(\lambda)$ (where $\lambda$ is the security parameter). Using the techniques from [14/1|11], it is possible to encrypt the secret key in a "packed" form, hence reducing the number of ciphertexts to $O(\log \lambda)$ (so we can get public keys of size quasi-linear in $\lambda$ ). Using our technique from this work, it is even possible to store an encryption of the secret key as a single ciphertext, as described in Section 4 We next outline our main bootstrapping technique in a few more details.

Our method applies mainly to "leveled" schemes that use the noise control mechanism of Brakerski-Gentry-Vaikuntanathan [1] 2 Below and throughout this paper we concentrate on the BGV ring-LWE-based scheme, since it offers the most efficient

\footnotetext{
${ }^{2}$ Our method can be used also with other schemes, as long as the scheme allows us to choose a modulus very close to a power of two. For example they can be used with the schemes from [32].
} 
homomorphic operations and the most room for optimizations 3 The scheme is defined over a ring $R=\mathbb{Z}[X] / F(X)$ for a monic, irreducible polynomial $F(X)$ (over the integers $\mathbb{Z}$ ). For an arbitrary integer modulus $n$ (not necessarily prime) we denote the ring $R_{n} \stackrel{\text { def }}{=} R / n R=(\mathbb{Z} / n \mathbb{Z})[X] / F(X)$. The scheme is parametrized by the number of levels that it can handle, which we denote by $L$, and by a set of decreasing odd moduli $q_{0} \gg q_{1} \gg \cdots \gg q_{L}$, one for each level.

The plaintext space is given by the ring $R_{2}$, while the ciphertext space for the $i$ 'th level consists of vectors in $\left(R_{q_{i}}\right)^{2}$. Secret keys are polynomials $\mathfrak{s} \in R$ with "small" coefficients, and we view $\mathfrak{s}$ as the second element of the 2 -vector $\mathbf{s}=(1, \mathfrak{s})$. A level$i$ ciphertext $\mathbf{c}=\left(c_{0}, c_{1}\right)$ encrypts a plaintext polynomial $m \in R_{2}$ with respect to $\mathbf{s}=(1, \mathfrak{s})$ if we have the equality over $R,[\langle\mathbf{c}, \mathbf{s}\rangle]_{q_{i}}=\left[c_{0}+\mathfrak{s} \cdot c_{1}\right]_{q_{i}} \equiv m(\bmod 2)$, and moreover the polynomial $\left[c_{0}+\mathfrak{s} \cdot c_{1}\right]_{q_{i}}$ is "small", i.e. all its coefficients are considerably smaller than $q_{i}$. Roughly, that polynomial is considered the "noise" in the ciphertext, and its coefficients grow as homomorphic operations are performed 4 The crux of the noise-control technique from [1] is that a level- $i$ ciphertext can be publicly converted into a level- $(i+1)$ ciphertext (with respect to the same secret key), and that this transformation reduces the noise in the ciphertext roughly by a factor of $q_{i+1} / q_{i}$.

Secret keys too are associated with levels, and the public key includes some additional information that (roughly speaking) makes it possible to convert a ciphertext with respect to level- $i$ key $\mathbf{s}_{i}$ into a ciphertext with respect to level- $(i+1)$ key $\mathbf{s}_{i+1}$. In what follows we will only be interested in the secret keys at level $L$ and level zero; which we will denote by $\mathbf{s}$ and $\tilde{\mathbf{s}}$ respectively to ease notation.

For bootstrapping, we have as input a level- $L$ ciphertext (i.e. a vector $\mathbf{c} \in R / q_{L} R$ modulo the smallest modulus $q_{L}$ ). This means that the noise-control technique can no longer be applied to reduce the noise, hence (essentially) no more homomorphic operations can be performed on this ciphertext. To enable further computation, we must therefore "recrypt" the ciphertext $\mathbf{c}$, to obtain a new ciphertext that encrypts the same element of $R$ with respect to some lower level $i<L$.

Our first observation is that the decryption at level $L$ can be made more efficient when $q_{L}$ is close to a power of two, specifically $q_{L}=2^{r}+1$ for an integer $r$, and moreover the coefficients of $Z=\langle\mathbf{c}, \mathbf{s}\rangle \bmod F(X)$ are much smaller than $q_{L}^{2}$ in magnitude. In particular if $z$ is one of the coefficients of the polynomial $Z$ then $\left[[z]_{q_{L}}\right]_{2}$ can be computed as $z\langle r\rangle \oplus z\langle 0\rangle$, where $z\langle i\rangle$ is the $i$ 'th bit of $z$.

To evaluate the decryption formula homomorphically, we temporarily extend the plaintext space to polynomials modulo $2^{r+1}$ (rather than modulo 2 ). The level- $L$ secret key is $\mathbf{s}=(1, \mathfrak{s})$, where all the coefficients of $\mathfrak{s}$ are small (in the interval $\left(-2^{r},+2^{r}\right)$ ). We can therefore consider $\mathfrak{s}$ as a plaintext polynomial in $R / 2^{r+1} R$, encrypt it inside a level-0 ciphertext, and keep that ciphertext in the public key. Thus, given the level- $L$ ciphertext $\mathbf{c}$, we can evaluate the inner product $[\langle\mathbf{c}, \mathbf{s}\rangle \bmod F(X)]$ homomorphically, obtaining a level- 0 ciphertext that encrypts the polynomial $Z$.

For simplicity, assume for now that what we get is an encryption of all the coefficients of $Z$ separately. Given an encryption of a coefficient $z$ of $Z$ (which is an element

\footnotetext{
${ }^{3}$ Our description of the BGV cryptosystem below assumes modulo-2 plaintext arithmetic, generalizing to modulo- $p$ arithmetic for other primes $p>2$ is straightforward.

${ }^{4}$ We ignore here the encryption procedure, since it does not play any role in the current work.
} 
in $\mathbb{Z} / 2^{r+1} \mathbb{Z}$ ) we show in Section 3.1 how to extract (encryptions of) the zero'th and $r$ 'th bit using a data-oblivious algorithm. Hence we can finally recover a new ciphertext, encrypting the same binary polynomial at a lower level $i<L$.

To achieve efficient bootstrapping, we exploit the ability to perform operations on elements modulo $2^{r+1}$ in a SIMD fashion (Single Instruction Multiple $\underline{\text { Data); }}$; much like in prior work [14]1|11]. Some care must be taken when applying these techniques in our case, since the inputs and outputs of the bootstrapping procedure are not in the correct format: Specifically, these techniques require that inputs and outputs be represented using polynomial Chinese Remainders (CRT representation), whereas decryption (and therefore recryption) inherently deals with polynomials in coefficient representation. We therefore must use explicit conversion to CRT representation, and ensure that these conversions are efficient enough. See details in Section 4

Also, the techniques from prior work must be extended somewhat to be usable in our case: Prior work demonstrated that SIMD operations can be performed homomorphically when the underlying arithmetic is over a field, but in our case we have operations over the ring $\mathbb{Z} / 2^{r+1} \mathbb{Z}$, which is not a field. The algebra needed to extend the SIMD techniques to this case is essentially an application of the theory of local fields [4]. We prove many of the basic results that we need in the full version [10], and refer the reader to [4] for a general introduction and more details.

Notations. Throughout the paper we denote by $[z]_{q}$ the reduction of $z \bmod q$ into the interval $\left(-\frac{q}{2}, \frac{q}{2}\right]$. We also denote the $i$ 'th bit in the binary representation of the integer $z$ by $z\langle i\rangle$. Similarly, when $a$ is an integer polynomial of degree $d$ with coefficients $\left(a_{0}, a_{1}, \ldots, a_{d}\right)$, we denote by $a\langle i\rangle$ the $0-1$ degree- $d$ polynomial whose coefficients are all the $i$ 'th bits $\left(a_{0}\langle i\rangle, a_{1}\langle i\rangle, \ldots, a_{d}\langle i\rangle\right)$. If $\mathbf{c}, \mathbf{s}$ are two same-dimension vectors, then $\langle\mathbf{c}, \mathbf{s}\rangle$ denotes their inner product.

Organization. We begin by presenting the simplified decryption formula in Section 2 and explain how to evaluate it homomorphically in Section 3 Then in Section 4 we recall some algebra and explain how to use techniques similar to [11] to run bootstrapping in time quasi-linear in the security parameter. Some of the proofs are omitted here, these are found in the full version of this work [10].

\section{A Simpler Decryption Formula}

When the small modulus $q_{L}$ has a special form -i.e. when it equals $u \cdot 2^{r}+v$ for some integer $r$ and for some small positive odd integers $u, v$ - then the mod- $q_{L}$ decryption formula can be made to have a particularly simple form. Below we focus on the case of $q_{L}=2^{r}+1$, which suffices for our purposes.

So, assume that $q_{L}=2^{r}+1$ for some integer $r$ and that we decrypt by setting $a \leftarrow\left[[\langle\mathbf{c}, \mathbf{s}\rangle \bmod F(X)]_{q_{L}}\right]_{2}$. Consider now the coefficients of the integer polynomial $Z=\langle\mathbf{c}, \mathbf{s}\rangle \bmod F(X)$, without the reduction $\bmod q_{L}$. Since $\mathbf{s}$ has small coefficients (and we assume that reduction mod- $F(X)$ does not increase the coefficients by much) then all the coefficients of $Z$ are much smaller than $q_{L}^{2}$. Consider one of these integer coefficients, denoted by $z$, so we know that $|z| \ll q_{L}^{2} \approx 2^{2 r}$. We consider the binary 
representation of $z$ as a $2 r$-bit integer, and assume for now that $z \geq 0$ and also $[z]_{q_{L}} \geq$ 0 . We claim that in this case, the bit $\left[[z]_{q_{L}}\right]_{2}$ can be computed simply as the sum of the lowest bit and the $r$ 'th bit of $z$, i.e., $\left[[z]_{q_{L}}\right]_{2}=z\langle r\rangle \oplus z\langle 0\rangle$. (Recall that $z\langle i\rangle$ is the $i$ 'th bit of $z$.)

Lemma 1. Let $q=2^{r}+1$ for a positive integer $r$, and let $z$ be a non-negative integer smaller than $\frac{q^{2}}{2}-q$, such that $[z]_{q}$ is also non-negative, $[z]_{q} \in\left[0, \frac{q}{2}\right]$. Then $\left[[z]_{q}\right]_{2}=$ $z\langle r\rangle \oplus z\langle 0\rangle$.

Proof. Let $z_{0}=[z]_{q} \in\left[0, \frac{q}{2}\right]$, and consider the sequence of integers $z_{i}=z_{0}+i q$ for $i=0,1,2, \cdots$. Since we assume that $z \geq 0$ then $z$ can be found in this sequence, say the $k$ 'th element $z=z_{k}=z_{0}+k q$. Also since $z<\frac{q^{2}}{2}-q$ then $k=\lfloor z / q\rfloor<\frac{q}{2}-1$. The bit that we want to compute is $\left[[z]_{q}\right]_{2}=z_{0}\langle 0\rangle$. We claim that $z_{0}\langle 0\rangle=z_{k}\langle 0\rangle+z_{k}\langle r\rangle$ $(\bmod 2)$. This is because $z_{k}=z_{0}+k q=z_{0}+k\left(2^{r}+1\right)=\left(z_{0}+k\right)+k 2^{r}$, which in particular means that $z_{k}\langle 0\rangle=z_{0}\langle 0\rangle+k\langle 0\rangle(\bmod 2)$. But since $0 \leq z_{0} \leq q / 2$ and $0 \leq k<q / 2-1$ then $0 \leq z_{0}+k<q-1=2^{r}$, so there is no carry bit from the addition $z_{0}+k$ to the $r$ 'th bit position. It follows that the $r$ 'th bit of $z_{k}$ is equal to the 0'th bit of $k$ (i.e., $z_{k}\langle r\rangle=k\langle 0\rangle$ ), and therefore $z_{k}\langle 0\rangle=z_{0}\langle 0\rangle+k\langle 0\rangle=z_{0}\langle 0\rangle+z_{k}\langle r\rangle$ $(\bmod 2)$, which implies that $z_{0}\langle 0\rangle=z_{k}\langle 0\rangle+z_{k}\langle r\rangle(\bmod 2)$, as needed.

We note that the proof can easily be extended for the case $q=u 2^{r}+v$, if the bound on $z$ is strengthened by a factor of $v$. To remove the assumption that both $z$ and $[z]_{q}$ are non-negative, we use the following easy corollary:

Corollary 1. Let $r \geq 3$ and $q=2^{r}+1$ and let $z$ be an integer with absolute value smaller than $\frac{q^{2}}{4}-q$, such that $[z]_{q} \in\left(-\frac{q}{4}, \frac{q}{4}\right)$. Then $\left[[z]_{q}\right]_{2}=z\langle r\rangle \oplus z\langle r-1\rangle \oplus z\langle 0\rangle$.

Proof. Denoting $z^{\prime}=z+\left(q^{2}-1\right) / 4=z+(q+1)(q-1) / 4=\left(z+\frac{q-1}{4}\right)+q \cdot \frac{q-1}{4}$, we have $z^{\prime} \equiv z+\frac{q-1}{4}(\bmod q)$ (since $\frac{q-1}{4}=2^{r-2}$ is an integer). Moreover since $[z]_{q} \in$ $\left(-\frac{q}{4}, \frac{q}{4}\right]$ then $[z]_{q}+\frac{q-1}{4} \in[0, q / 2]$, hence $\left[z^{\prime}\right]_{q}=[z]_{q}+\frac{q-1}{4}$ (over the integers), and as $\frac{q-1}{4}$ is an even integer then $[z]_{q}=\left[z^{\prime}\right]_{q}(\bmod 2)$, or in other words $\left[[z]_{q}\right]_{2}=\left[\left[z^{\prime}\right]_{q}\right]_{2}$. Since $z>-\frac{q^{2}}{4}$ and $z$ is an integer then $z \geq-\frac{q^{2}-1}{4}$ and therefore $z^{\prime}=z+\frac{q^{2}-1}{4} \geq 0$. Thus $z^{\prime}$ satisfies all the conditions set in Lemma 1 so applying that lemma we have $\left[[z]_{q}\right]_{2}=\left[\left[z^{\prime}\right]_{q}\right]_{2}=z^{\prime}\langle r\rangle \oplus z^{\prime}\langle 0\rangle$.

We next observe that $z^{\prime}=z+(q+1)(q-1) / 4=z+\left(2^{r}+2\right) 2^{r-2}=z+2^{r-1}+2^{2 r-2}$. Since $2 r-2>r$, this means that the bits 0 through $r$ in the binary representation of $z^{\prime}$ are determined by $z+2^{r-1}$ alone, so we have:

$$
\begin{aligned}
z^{\prime}\langle i\rangle & =z\langle i\rangle \text { for } i=0,1, \ldots, r-2 \\
z^{\prime}\langle r-1\rangle & =1-z\langle r-1\rangle \\
z^{\prime}\langle r\rangle & =\left\{\begin{array}{ll}
z\langle r\rangle & \text { if } z\langle r-1\rangle=0 \\
1-z\langle r\rangle & \text { if } z\langle r-1\rangle=1
\end{array}\right\}=z\langle r\rangle \oplus z\langle r-1\rangle
\end{aligned}
$$

Putting it all together, we get $\left[[z]_{q}\right]_{2}=\left[\left[z^{\prime}\right]_{q}\right]_{2}=z^{\prime}\langle r\rangle \oplus z^{\prime}\langle 0\rangle=z\langle r\rangle \oplus z\langle r-1\rangle \oplus z\langle 0\rangle$. 
Using Corollary 1 we can get our simplified decryption formula. First, we set our parameters such that $q_{L}=2^{r}+1$ and all the coefficients of the integer polynomial $Z=\langle\mathbf{c}, \mathbf{s}\rangle \bmod F(X)$ are smaller than $\frac{q_{L}^{2}}{4}-1$ in absolute value, and moreover they are all less than $\frac{q_{L}-1}{4}$ away from a multiple of $q_{L}$. Given a two-element ciphertext $\mathbf{c}=\left(c_{0}, c_{1}\right) \in\left(\left(\mathbb{Z} / q_{L} \mathbb{Z}\right)[X] / F(X)\right)^{2}$, then compute $Z \leftarrow\langle\mathbf{c}, \mathbf{s}\rangle \bmod F(X)$ over the integers (without reduction $\bmod q_{L}$ ), and finally recover the plaintext as $Z\langle r\rangle+$ $Z\langle r-1\rangle+Z\langle 0\rangle$. Ultimately, we obtain the plaintext polynomial $a \in \mathbb{F}_{2}[X] / F(X)$, where each coefficient in $a$ is obtained as the XOR of bits $0, r-1$, and $r$ of the corresponding coefficient in $Z$.

Working Modulo $2^{r+1}$. Since we are only interested in the contents of bit positions $0, r-1$, and $r$ in the polynomial $Z$, we can compute $Z$ modulo $2^{r+1}$ rather than over the integers. Our simplified decryption of a ciphertext vector $\mathbf{c}=\left(c_{0}, c_{1}\right)$ proceeds as follows:

1. Compute $Z \leftarrow[\langle\mathbf{c}, \mathbf{s}\rangle \bmod F(X)]_{2^{r+1}}$;

2. Recover the 0-1 plaintext polynomial $a=[Z\langle r\rangle+Z\langle r-1\rangle+Z\langle 0\rangle]_{2}$.

\section{Basic Homomorphic Decryption}

To get a homomorphic implementation of the simplified decryption formula from above, we use an instance of our homomorphic encryption scheme with underlying plaintext space $\mathbb{Z}_{2^{r+1}}$. Namely, denoting by $\tilde{\mathbf{s}}$ the level- 0 secret-key and by $q_{0}$ the largest modulus, a ciphertext encrypting $a \in\left(\mathbb{Z} / 2^{r+1} \mathbb{Z}\right)[X] / F(X)$ with respect to $\tilde{\mathbf{s}}$ and $q_{0}$ is a 2 -vector $\tilde{\mathbf{c}}$ over $\left(\mathbb{Z} / q_{0} \mathbb{Z}\right)[X] / F(X)$ such that $\left|[\langle\tilde{\mathbf{c}}, \tilde{\mathbf{s}}\rangle \bmod F(X)]_{q_{0}}\right| \ll q_{0}$ and $[\langle\tilde{\mathbf{c}}, \tilde{\mathbf{s}}\rangle \bmod F(X)]_{q_{0}} \equiv a\left(\bmod 2^{r+1}\right)$.

Recall that the ciphertext before bootstrapping is with respect to secret key $\mathbf{s}$ and modulus $q_{L}=2^{r}+1$. In this section we only handle the simple case where the public key includes an encryption of each coefficient of the secret-key s separately. Namely, denoting $\mathbf{s}=(1, \mathfrak{s})$ and $\mathfrak{s}(X)=\sum_{j=0}^{d-1} \mathfrak{s}_{j} X^{j}$, we encode for each $j$ the coefficient $\mathfrak{s}_{j}$ as the constant polynomial $\mathfrak{s}_{j} \in\left(\mathbb{Z} / 2^{r+1} \mathbb{Z}\right)[X] / F(X)$. (I.e., the degree- $d$ polynomial whose free term is $\mathfrak{s}_{j} \in\left[-2^{r}+1,2^{r}\right]$ and all the other coefficients are zero.) Then for each $j$ we include in the public key a ciphertext $\tilde{\mathbf{c}}_{j}$ that encrypts this constant polynomial $\mathfrak{s}_{j}$ with respect to $\tilde{\mathbf{s}}$ and $q_{0}$. Below we abuse notations somewhat, using the same notation to refer both to a constant polynomial $z \in\left(\mathbb{Z} / 2^{r} \mathbb{Z}\right)[X] / F(X)$ and the free term of that polynomial $z \in\left(\mathbb{Z} / 2^{r} \mathbb{Z}\right)$.

Computing Z Homomorphically. Given the $q_{L}$-ciphertext $\mathbf{c}=\left(c_{0}, c_{1}\right)$ (that encrypts a plaintext polynomial $\left.a \in \mathbb{F}_{2}[X] / F(X)\right)$, we use the encryption of $\mathbf{s}$ from the public key to compute the simple decryption formula from above. Computing an encryption of $Z=[\langle\mathbf{c}, \mathbf{s}\rangle \bmod F(X)]_{2^{r+1}}$ is easy, since the coefficients of $Z$ are just affine functions (over $\left(\mathbb{Z} / 2^{r+1} \mathbb{Z}\right)$ ) of the coefficients of $\mathfrak{s}$, which we can compute from the encryption of the $\mathfrak{s}_{j}$ 's in the public key. 


\subsection{Extracting the Top and Bottom Bits}

Now that we have encryptions of the coefficients of $Z$, we need to extract the relevant three bits in each of these coefficients and add them (modulo 2) to get encryptions of the plaintext coefficients. In more details, given a ciphertext $\tilde{\mathbf{c}}$ satisfying $[\langle\tilde{\mathbf{c}}, \tilde{\mathbf{s}}\rangle \bmod$ $F(X)]_{q_{0}} \equiv z\left(\bmod 2^{r+1}\right)$ where $z$ is some constant polynomial, we would like to compute another ciphertext $\tilde{\mathbf{c}}$ satisfying $[\langle\tilde{\mathbf{c}}, \tilde{\mathbf{s}}\rangle \bmod F(X)]_{q_{0}} \equiv z\langle 0\rangle+z\langle r-1\rangle+z\langle r\rangle$ $(\bmod 2)\left(\right.$ with $[\langle\tilde{\mathbf{c}}, \tilde{\mathbf{s}}\rangle \bmod F(X)]_{q_{0}}$ still much smaller then $q_{0}$ in magnitude). To this end, we describe a procedure to compute for all $i=0,1, \ldots, r$ a ciphertext $\tilde{\mathbf{c}}_{i}$ satisfying $\left[\left\langle\tilde{\mathbf{c}}_{i}, \tilde{\mathbf{s}}\right\rangle \bmod F(X)\right]_{q_{0}} \equiv z\langle i\rangle(\bmod 2)$. Clearly, we can immediately set $\tilde{\mathbf{c}}_{0}=\tilde{\mathbf{c}}$, we now describe how to compute the other $\tilde{\mathbf{c}}_{i}$ 's.

The basic observation underlying this procedure is that modulo a power of 2 , the second bit of $z-z^{2}$ is the same as that of $z$, but the LSB is zero-ed out. Thus setting $z^{\prime}=\left(z-z^{2}\right) / 2$ (which is an integer), we get that the LSB of $z^{\prime}$ is the second bit of $z$. More generally, we have the following lemma:

Lemma 2. Let $z$ be an integer with binary representation $z=\sum_{i=0}^{r} 2^{i} z\langle i\rangle$. Define $w_{0} \stackrel{\text { def }}{=} z$, and for $i \geq 1$ define

$$
w_{i} \stackrel{\text { def }}{=} \frac{z-\sum_{j=0}^{i-1} 2^{j} w_{j}^{2^{i-j}} \bmod 2^{r+1}}{2^{i}} \text { (division by } 2^{i} \text { over the rationals). }
$$

Then the $w_{i}$ 's are integers and we have $w_{i}\langle 0\rangle=z\langle i\rangle$ for all $i$.

Proof. The lemma clearly holds for $i=0$. Now fix some $i \geq 1$, assume that the lemma holds for all $j<i$, and we prove that it holds also for $i$. It is easy to show by induction that for any integer $u$ and all $j \leq r$ we have

$$
u^{2^{j}} \bmod 2^{r+1}=u\langle 0\rangle+2^{j+1} t \text { for some integer } t .
$$

Namely, the LSB of $u^{2^{j}} \bmod 2^{r+1}$ is the same as the LSB of $u$, and the next $j$ bits are all zero. This means that the bit representation of $v_{j} \stackrel{\text { def }}{=} 2^{j} w_{j}^{2^{i-j}} \bmod 2^{r+1}$ has bits $0,1, \ldots, j-1$ all zero (due to the multiplication by $2^{j}$ ), then $v_{j}\langle j\rangle=w_{j}\langle 0\rangle=z\langle j\rangle$ (by the induction hypothesis), and the next $i-j$ bits are again zero (by the observation above). In other words, the lowest $i+1$ bits of $v_{j}$ are all zero, except the $j$ 'th bit which is equal to the $j$ 'th bit of $z$.

This means that the lowest $i$ bits of the sum $\sum_{j=0}^{i-1} v_{j}$ are the same as the lowest $i$ bits of $z$, and the $i+1$ 'st bit of the sum is zero. Hence the lowest $i$ bits of $z-\sum_{j=0}^{i-1} v_{j}$ are all zero, and the $i+1$ 'st bit is $z\langle i\rangle$. Hence $z-\sum_{j=0}^{i-1} v_{j}$ is divisible by $2^{i}$ (over the integers), and the lowest bit of the result is $z\langle i\rangle$, as needed.

Our procedure for computing the ciphertexts $\tilde{\mathbf{c}}_{i}$ mirrors Lemma 2 . Specifically, we are given the ciphertext $\tilde{\mathbf{c}}=\tilde{\mathbf{c}}_{0}$ that encrypts $z=w_{0} \bmod 2^{r+1}$, and we iteratively compute ciphertexts $\tilde{\mathbf{c}}_{1}, \tilde{\mathbf{c}}_{2}, \ldots$ such that $\tilde{\mathbf{c}}_{i}$ encrypts $w_{i} \bmod 2^{r-i+1}$. Eventually we get $\tilde{\mathbf{c}}_{r}$ that encrypts $w_{r}$ mod 2, which is what we need (since the LSB of $w_{r}$ is the $r$ 'th bit of $z$ ). 
Note that most of the operations in Lemma 2 are carried out in $\left(\mathbb{Z} / 2^{r+1} \mathbb{Z}\right)$, and therefore can be evaluated homomorphically in our $\left(\mathbb{Z} / 2^{r+1} \mathbb{Z}\right)$-homomorphic cryptosystem. The only exception is the division by $2^{i}$ in Equation (1), and we now show how this division can also be evaluated homomorphically. To implement division we begin with an arbitrary ciphertext vector $\tilde{\mathbf{c}}$ that encrypts a plaintext element $a \in\left(\mathbb{Z} / 2^{j} \mathbb{Z}\right)[X] / F(X)$ (for some $j$ ) with respect to the level-0 key $\tilde{\mathbf{s}}$ and modulus $q_{0}$. Namely, we have the equality over $\mathbb{Z}[X]$ :

$$
(\langle\tilde{\mathbf{c}}, \tilde{\mathbf{s}}\rangle \bmod F(X))=a+2^{j} \cdot S+q_{0} \cdot T
$$

for some polynomials $S, T \in \mathbb{Z}[X] / F(X)$, where the norm of $a+2^{j} S$ is much smaller than $q_{0}$. Assuming that $a$ is divisible by 2 over the integers (i.e., all its coefficients are even) consider what happens when we multiply $\tilde{\mathbf{c}}$ by the integer $\left(q_{0}+1\right) / 2$ (which is the inverse of 2 modulo $q_{0}$ ). Then we have

$$
\begin{aligned}
\left(\left\langle\frac{q_{0}+1}{2} \cdot \tilde{\mathbf{c}}, \tilde{\mathbf{s}}\right\rangle \bmod F(X)\right)=\frac{q_{0}+1}{2} \cdot(\langle\tilde{\mathbf{c}}, \tilde{\mathbf{s}}\rangle \bmod F(X)) \\
\quad=\frac{\left(q_{0}+1\right) \cdot a}{2}+\frac{\left(q_{0}+1\right) \cdot 2^{j} \cdot S}{2}+\frac{q_{0} \cdot\left(q_{0}+1\right) \cdot T}{2} \\
\quad=\left(q_{0}+1\right) \cdot(a / 2)+\left(q_{0}+1\right) \cdot 2^{j-1} S+q_{0} \cdot \frac{q_{0}+1}{2} \cdot T \\
\quad=a / 2+2^{j-1} \cdot S+q_{0} \cdot\left(a / 2+2^{j-1} S+\frac{q_{0}+1}{2} T\right)
\end{aligned}
$$

Clearly the coefficients of $a / 2+2^{j-1} S$ are half the size of those of $a+2^{j} S$, hence they are much smaller than $q_{0}$. It follows that $\tilde{\mathbf{c}}^{\prime}=\left[\tilde{\mathbf{c}} \cdot\left(q_{0}+1\right) / 2\right]_{q_{0}}$ is a valid ciphertext that encrypts the plaintext $a / 2 \in\left(\mathbb{Z} / 2^{j-1} \mathbb{Z}\right)[X] / F(X)$ with respect to secret key $\tilde{\mathbf{s}}$ and modulus $q_{0}$.

The same argument shows that if $a$ is divisible by $2^{i}$ over the integers (for some $i<j$ ) then $\left[\tilde{\mathbf{c}} \cdot\left(\left(q_{0}+1\right) / 2\right)^{i}\right]_{q_{0}}$ is a valid ciphertext encrypting $a / 2^{i} \in\left(\mathbb{Z} / 2^{j-i} \mathbb{Z}\right)[X] / F(X)$. Combining this division-by-two procedure with homomorphic exponentiation mod $2^{r+1}$, the resulting homomorphic bit-extraction procedure is described in Figure 1.

\subsection{Packing the Coefficients}

Now that we have encryption of all the coefficients of $a$, we just need to "pack" all these coefficients back in one polynomial. Namely, we have encryption of the constant polynomials $a_{0}, a_{1}, \ldots$, and we want to get an encryption of the polynomial $a(X)=$ $\sum_{i} a_{i} X^{i}$. Since $a$ is just a linear combination of the $a_{i}$ 's (with the coefficient of each $a_{i}$ being the "scalar" $\left.X^{i} \in(Z / 2 Z)[X] / \Phi_{m}\right)$, we can just use the additive homomorphism of the cryptosystem to compute an encryption of $a$ from the encryptions of the $a_{i}$ 's.

\subsection{Lower-Degree Bit Extraction}

As described in Figure 1, extracting the $r$ 'th bit requires computing polynomials of degree upto $2^{r}$, here we describe a simple trick to lower this degree. Recall our simplified decryption process: we set $Z \leftarrow\left[\langle\mathbf{c}, \mathbf{s}\rangle \bmod \Phi_{m}(X)\right]_{2^{r+1}}$, and then recover $a=[Z\langle r\rangle+Z\langle r-1\rangle+Z\langle 0\rangle]_{2}$. 
$\operatorname{Bit-Extraction}\left(\tilde{\mathbf{c}}, r, q_{0}\right)$ :

Input: A ciphertext $\tilde{\mathbf{c}}$ encrypting a constant $b \in\left(\mathbb{Z} / 2^{r+1} \mathbb{Z}\right)$ w.r.t. secret key $\tilde{\mathbf{s}}$ and modulus $q_{0}$. Output: A ciphertext $\tilde{\mathbf{c}}^{\prime}$ encrypting $b\langle 0\rangle \oplus b\langle r-1\rangle \oplus b\langle r\rangle \in \mathbb{F}_{2}$ w.r.t. secret key $\tilde{\mathbf{s}}$ and modulus $q_{0}$.

1. Set $\tilde{\mathbf{c}}_{0} \leftarrow \tilde{\mathbf{c}}$

// $\tilde{\mathbf{c}}$ encrypt $z$ w.r.t. $\tilde{\mathbf{s}}$

2. For $i=1$ to $r$

3. $\quad$ Set $\operatorname{acc} \leftarrow \tilde{\mathbf{c}}$

$/ /$ acc is an accumulator

4. For $j=0$ to $i-1$

// Compute $z-\sum_{j} 2^{j} w_{j}^{i-1}$

5. Set $\operatorname{tmp} \leftarrow \operatorname{Hom} \operatorname{Exp}\left(\tilde{\mathbf{c}}_{j}, 2^{i-j}\right) \quad / /$ Homomorphic exponentiation to the power $2^{i-j}$

6. $\quad$ Set acc $\leftarrow$ acc $-2^{j} \cdot \mathbf{t m p} \bmod q_{0}$

7. Set $\tilde{\mathbf{c}}_{i} \leftarrow \mathbf{a c c} \cdot\left(\left(q_{0}+1\right) / 2\right)^{i} \bmod q_{0} \quad / / \tilde{\mathbf{c}}_{i}$ encrypts $z\langle i\rangle$

8. Output $\tilde{\mathbf{c}}_{0}+\tilde{\mathbf{c}}_{r-1}+\tilde{\mathbf{c}}_{r} \bmod q_{0}$

$\operatorname{Hom} \operatorname{Exp}(\tilde{\mathbf{c}}, n)$ uses native homomorphic multiplication to multiply $\tilde{\mathbf{c}}$ by itself $n$ times. To aid exposition, this code assumes that the modulus and secret key remain fixed, else modulus-switching and key-switching should be added (and the level increased correspondingly to some $i>0$ ).

Fig. 1. A Homomorphic Bit-Extraction Procedure

Consider what happens if we add $q_{L}$ to all the odd coefficients in c, call the resulting vector $\mathbf{c}^{\prime}$ : On one hand, now all the coefficients of $\mathbf{c}^{\prime}$ are even. On the other hand, the coefficients of $Z^{\prime}=\left\langle\mathbf{c}^{\prime}, \mathbf{s}\right\rangle \bmod \Phi_{m}(X)$ are still small enough to use Lemma1 (since they are at most $c_{m} \cdot q \cdot\|\mathbf{s}\|_{1}$ larger than those of $Z$ itself, where $c_{m}$ is the ring constant of mod- $\Phi_{m}(X)$ arithmetic and $\|s\|_{1}$ is the $l_{1}$-norm of $\left.\mathbf{s}\right)$. Since $\mathbf{c}^{\prime}=\mathbf{c}\left(\bmod q_{L}\right)$ then we have

$$
\left[\left[\langle\mathbf{c}, \mathbf{s}\rangle \bmod \Phi_{m}(X)\right]_{q_{L}}\right]_{2}=\left[\left[\left\langle\mathbf{c}^{\prime}, \mathbf{s}\right\rangle \bmod \Phi_{m}(X)\right]_{q_{L}}\right]_{2}=Z^{\prime}\langle r\rangle+Z^{\prime}\langle r\rangle-1+Z^{\prime}\langle 0\rangle
$$

However, since $\mathbf{c}^{\prime}$ is even then so is $Z^{\prime}$. This means that $Z^{\prime}\langle 0\rangle=0$, and if we divide $Z^{\prime}$ by two (over the integers), $Z^{\prime \prime}=Z^{\prime} / 2$, then we have $\left[\left[\langle\mathbf{c}, \mathbf{s}\rangle \bmod \Phi_{m}(X)\right]_{q_{L}}\right]_{2}=$ $Z^{\prime \prime}\langle r-1\rangle \oplus Z^{\prime \prime}\langle r-2\rangle$. We thus have a variation of the simple decryption formula that only needs to extract the $r-1$ 'st and $r-2$ 'nd bits, so it can be realized using polynomials of degree upto $2^{r-1}$. Note that we can implement this variant of the decryption formula homomorphically, because $Z^{\prime}$ is even so an $q_{0}$-encryption of $Z^{\prime}$ can be easily converted into an encryption of $Z^{\prime} / 2$ (by multiplying by $\frac{q_{0}+1}{2}$ modulo $q_{0}$ as described in Section 3.1.

This technique can be pushed a little further, adding to $\mathbf{c}$ multiples of $q$ so that it is divisible by $4,8,16$, etc., and reducing the required degree correspondingly to $2^{r-2}$, $2^{r-3}, 2^{r-4}$, etc. The limiting factor is that we must maintain that $\left\langle\mathbf{c}^{\prime}, \mathbf{s}\right\rangle$ has coefficients sufficiently smaller than $q_{L}^{2}$, in order to be able to use Lemma 1 Clearly, if $\mathbf{c}^{\prime}=\mathbf{c}+q \kappa$ where all the coefficients of $\kappa$ are smaller than some bound $B$ (in absolute value), then the coefficients of $\left\langle\mathbf{c}^{\prime}, \mathbf{s}\right\rangle$ can be larger than the coefficients of $Z=\langle\mathbf{c}, \mathbf{s}\rangle$ (in absolute value) by at most $c_{m} \cdot q \cdot B \cdot\|\mathbf{s}\|_{1}$. (Heuristically we expect the difference to depend on the $l_{2}$ norm of $\mathbf{s}$ more than its $l_{1}$ norm.)

If we choose our parameters such that the $l_{1}$-norm of $\mathbf{s}$ is below $m$, and work over a ring with $c_{m}=O(1)$, then the coefficients of $Z$ can be made as small as $c_{m} \cdot m \cdot q$, and we can make the coefficients of $\kappa$ as large as $B \approx q /\left(4 c_{m} \cdot m\right)$ in absolute value while maintaining the invariant that the coefficients of $Z^{\prime}$ are smaller than $q^{2} / 4$ (which 
is what we need to be able to use Lemma 1). By choosing an appropriate $\kappa$, we can ensure that the least significant $\left\lfloor\log \left(q /\left(4 c_{m} m\right)\right)\right\rfloor=r-\left\lceil\log \left(4 c_{m} m\right)\right\rceil$ bits of $\mathbf{c}^{\prime}$ are all zero. This means that we can implement bit extraction using only polynomials of degree at most $2^{\left\lceil\log \left(4 c_{m} m\right)\right\rceil}<8 c_{m} m=O(m)$. (Heuristically, we should even be able to get polynomials of degree $O(\sqrt{m})$ since the $l_{2}$ norm of $\mathbf{s}$ is only $O(\sqrt{m})$.) Moreover if we assume that ring-LWE is hard even with a very sparse secret, then we can use a secret key with even smaller norm and get the same reduction in the degree of the bit-extraction routine.

\section{Homomorphic Decryption with Packed Ciphertexts}

The homomorphic decryption procedure from Section 3 is rather inefficient, mostly because we need to repeat the bit-extraction procedure from Figure 1 for each coefficient separately. Instead, we would like to pack many coefficients in one ciphertext and extract the top bits of all of them together. To this end we employ a batching technique, similar to [1]11 14], using Chinese remaindering over the ring of polynomials to pack many "plaintext slots" inside a single plaintext polynomial.

Recall that the BGV scheme is defined over a polynomial ring $R=\mathbb{Z}[X] / F(X)$. If the polynomial $F(X)$ factors modulo two into distinct irreducible polynomials $F_{0}(X) \times \cdots \times F_{\ell-1}(X)$, then, by the Chinese Remainder Theorem, the plaintext space factors into a product of finite fields $R_{2} \cong \mathbb{F}_{2}[X] / F_{0}(X) \times \cdots \times \mathbb{F}_{2}[X] / F_{\ell-1}(X)$.

This factorization is used in [141|11] to "pack" a vector of $\ell$ elements (one from each $\mathbb{F}_{2}[X] / F_{i}(X)$ ) into one plaintext polynomial, which is then encrypted in one ciphertext; each of the $\ell$ components called a plaintext slot. The homomorphic operations (add/mult) are then applied to the different slots in a SIMD fashion. When $F(X)$ is the $m$-th cyclotomic polynomial, $F(X)=\Phi_{m}(X)$, then the field $\mathbb{Q}[X] / F(X)$ is Galois (indeed Abelian) and so the polynomials $F_{i}(X)$ all have the same degree (which we will denote by $d$ ). It was shown in [11] how to evaluate homomorphically the application of the Galois group on the slots, and in particular this enables homomorphically performing arbitrary permutations on the vector of slots in time quasi-linear in $m$. This, in turn, is used in [11] to evaluate arbitrary arithmetic circuits (of average width $\tilde{\Omega}(\lambda)$ ) with overhead only polylog $(\lambda)$.

However, the prior work only mentions the case of plaintext spaces taken modulo a prime (in our case two), i.e. $R_{2}$. In this work we will need to also consider plaintext spaces which are given by a power of a prime, i.e. $R_{2^{r+1}}$ for some positive integer $r$. (We stress that by $R_{2^{t}}$ we really do mean $\left(\mathbb{Z} / 2^{t} \mathbb{Z}\right)[X] / F(X)$ and not $\mathbb{F}_{2^{t}}[X] / F(X)$.) In the full version [10] we show how the techniques from [11] extends also to this case. The "high brow" way of seeing this is to consider the message space modulo $2^{r+1}$ as the precision $r+1$ approximation to the 2-adic integers; namely we need to consider the localization of the field $K=\mathbb{Q}[X] / F(X)$ at the prime 2 .

\subsection{Using SIMD Techniques for Bootstrapping}

Using the techniques from [11] for bootstrapping is not quite straightforward, however. The main difficulty is that the input and output of are not presented in a packed form: 
The input is a single $q_{L}$-ciphertext that encrypts a single plaintext polynomial $a$ (which may or may not have many plaintext elements packed in its slots), and similarly the output needs to be a single ciphertext that encrypts the same polynomial $a$, but with respect to a larger modulus. (We stress that this is not an artifact of our "simpler decryption formula", we would need to overcome the same difficulty also if we tried to use these "SIMD techniques" to speed up bootstrapping under the standard approach of emulating the binary mod- $q_{L}$ circuit.) Our "packed bootstrapping" procedure consists of the following steps:

1. Using the encryption of the $q_{L}$-secret-key with respect to the modulus $q_{0}$, we convert the initial $q_{L}$-ciphertext into a $q_{0}$-ciphertext encrypting the polynomial $Z \in$ $\left(\mathbb{Z} / 2^{r+1} \mathbb{Z}\right)[X] / \Phi_{m}(X)$

2. Next we apply a homomorphic inverse-DFT transformation to get encryption of polynomials that have the coefficients of $Z$ in their plaintext slots.

3. Now that we have the coefficients of $Z$ in the plaintext slots, we apply the bit extraction procedure to all these slots in parallel. The result is encryption of polynomials that have the coefficients of $a$ in their plaintext slots.

4. Finally, we apply a homomorphic DFT transformation to get back a ciphertext that encrypts the polynomial $a$ itself.

Below we describe each of these steps in more detail. We note that the main challenge is to get an efficient implementation of Steps 2 and 4

\subsection{Encrypting the $q_{L}$-Secret-Key}

As in Section 3, we use an encryption scheme with underlying plaintext space modulo $2^{r+1}$ to encrypt the $q_{L}$-secret-key $\mathbf{s}$ under the $q_{0}$-secret-key $\tilde{\mathbf{s}}$. The $q_{L}$-secret-key is a vector $\mathbf{s}=(1, \mathfrak{s})$, where $\mathfrak{s} \in \mathbb{Z}[X] / \Phi_{m}(X)$ is an integer polynomial with small coefficients. Viewing these small coefficients as elements in $\mathbb{Z} / 2^{r+1} \mathbb{Z}$, we encrypt $\mathfrak{s}$ as a $q_{0}$-ciphertext $\tilde{\mathbf{c}}=\left(\tilde{\mathfrak{c}}_{0}, \tilde{\mathfrak{c}}_{1}\right)$ with respect to the $q_{0}$-secret-key $\tilde{\mathbf{s}}=(1, \tilde{\mathfrak{s}})$, namely we have

$$
\left.\left[\langle\tilde{\mathbf{c}}, \tilde{\mathbf{s}}\rangle \bmod \Phi_{m}\right]_{q_{0}}=\left[\tilde{\mathfrak{c}}_{0}+\tilde{\mathfrak{c}}_{1} \cdot \tilde{\mathfrak{s}} \bmod \Phi_{m}\right]_{q_{0}}=2^{r+1} \tilde{k}+\mathbf{s} \quad \text { (equality over } \mathbb{Z}[X]\right)
$$

for some polynomial $\tilde{k}$ with small coefficients.

\subsection{Step One: Computing Z Homomorphically}

Given a $q_{L}$-ciphertext $\mathbf{c}=\left(c_{0}, c_{1}\right)$ we recall from the public key the $q_{0}$ ciphertext $\tilde{\mathbf{c}}=$ $\left(\tilde{\mathfrak{c}}_{0}, \tilde{\mathfrak{c}}_{1}\right)$ that encrypts $\mathfrak{s}$, then compute the mod- $2^{r+1}$ inner product homomorphically by setting

$$
\tilde{\mathbf{z}}=\left(\left[c_{0}+c_{1} \tilde{\mathfrak{c}}_{0} \bmod \Phi_{m}\right]_{q_{0}}, \quad\left[c_{1} \tilde{\mathfrak{c}}_{1} \bmod \Phi_{m}\right]_{q_{0}}\right) .
$$

We claim that $\tilde{\mathbf{z}}$ is a $q_{0}$-ciphertext encrypting our $Z$ with respect to the secret key $\tilde{\mathbf{s}}$ (and plaintext space modulo $2^{r+1}$ ). To see that, recall that we have the following two equalities over $\mathbb{Z}[X]$,

$$
\left(c_{0}+c_{1} \mathfrak{s} \bmod \Phi_{m}\right)=2^{r+1} k+Z \text { and }\left(\tilde{\mathfrak{c}}_{0}+\tilde{\mathfrak{c}}_{1} \tilde{\mathfrak{s}} \bmod \Phi_{m}\right)=q_{0} \tilde{k}+2^{r+1} \tilde{k}^{\prime}+\mathfrak{s},
$$


where $k, \tilde{k}, \tilde{k}^{\prime} \in \mathbb{Z}[X] / \Phi_{m}$, the coefficients of $2^{r+1} k+Z$ are smaller than $2 q_{L}^{2} \ll q_{0}$, and the coefficients of $2^{r+1} \tilde{k}^{\prime}+\mathfrak{s}$ are also much smaller than $q_{0}$. It follows that:

$$
\begin{aligned}
(\langle\tilde{\mathbf{z}}, \tilde{\mathbf{s}}\rangle & \left.\bmod \Phi_{m}\right)=\left[c_{0}^{\prime}+c_{1} \tilde{\mathfrak{c}}_{0} \bmod \Phi_{m}\right]_{q_{0}}+\left(\tilde{\mathfrak{s}} \cdot\left[c_{1} \tilde{\mathfrak{c}}_{1} \bmod \Phi_{m}\right]_{q_{0}} \bmod \Phi_{m}\right) \\
& =\left(c_{0}^{\prime}+c_{1}\left(\tilde{\mathfrak{c}}_{0}+\tilde{\mathfrak{c}}_{1} \tilde{\mathfrak{s}}\right) \bmod \Phi_{m}\right)+q_{0} \kappa \\
& =\left(c_{0}^{\prime}+c_{1}\left(2^{r+1} \tilde{k}^{\prime}+\mathfrak{s}\right) \bmod \Phi_{m}\right)+q_{0} \kappa^{\prime} \\
& =\left(c_{0}^{\prime}+c_{1} \mathfrak{s} \bmod \Phi_{m}\right)+q_{0} \kappa^{\prime}+2^{r+1}\left(c_{1} \cdot \tilde{k}^{\prime} \bmod \Phi_{m}\right) \\
& \left.=q_{0} \kappa^{\prime}+2^{r+1}\left(k+c_{1} \tilde{k}^{\prime} \bmod \Phi_{m}\right)+Z \quad \text { (equality over } \mathbb{Z}[X]\right)
\end{aligned}
$$

for some $\kappa, \kappa^{\prime} \in \mathbb{Z}[X] / \Phi_{m}$. Moreover, since the coefficients of $c_{1}$ are smaller than $q_{L} \ll q_{0}$ then the coefficients of $2^{r+1}\left(k+c_{1} \tilde{k}^{\prime} \bmod \Phi_{m}\right)+Z$ are still much smaller than $q_{0}$. Hence $\tilde{\mathbf{z}}$ is decrypted under $\tilde{\mathbf{s}}$ and $q_{0}$ to $Z$, with plaintext space $2^{r+1}$.

\subsection{Step Two: Switching to CRT Representation}

Now that we have an encryption of the polynomial $Z$, we want to perform the homomorphic bit-extraction procedure from Figure 1. However, this procedure should be applied to each coefficient of $Z$ separately, which is not directly supported by the native homomorphism of our cryptosystem. (For example, homomorphically squaring the ciphertext yields an encryption of the polynomial $Z^{2} \bmod \Phi_{m}$ rather than squaring each coefficient of $Z$ separately.) We therefore need to convert $\tilde{\mathbf{z}}$ to CRT-based "packed" ciphertexts that hold the coefficients of $Z$ in their plaintext slots.

The system parameter $m$ was chosen so that $m=\tilde{\Theta}(\lambda)$ and $\Phi_{m}(X)$ factors modulo 2 (and therefore also modulo $2^{r+1}$ ) as a product of degree- $d$ polynomials with $d=O(\log m), \Phi_{m}(X)=\prod_{j=0}^{\ell-1} F_{j}(X)\left(\bmod 2^{r+1}\right)$. This allows us to view the plaintext polynomial $Z(X)$ as having $\ell$ slots, with the $j$ 'th slot holding the value $Z(X) \bmod$ $\left(F_{j}(X), 2^{r+1}\right)$. This way, adding/multipliying/squaring the plaintext polynomials has the effect of applying the same operation on each of the slots separately.

In our case, we have $\phi(m)$ coefficients of $Z(X)$ that we want to put in the plaintext slots, and each ciphertext has only $\ell=\phi(m) / d$ slots, so we need $d$ ciphertexts to holds them all. The transformation from the single ciphertext $\tilde{\mathbf{z}}$ that encrypts $Z$ itself to the collection of $d$ ciphertexts that hold the coefficients of $Z$ in their slots is described in Section 4.7 below. (We describe that step last, since it is the most complicated and it builds on machinery that we develop for Step Four in Section 4.6.)

\subsection{Step Three: Extracting the Relevant Bits}

Once we have the coefficients of $Z$ in the plaintext slots, we can just repeat the procedure from Figure 1. The input to the the bit-extraction procedure is a collection of some $d$ ciphertexts, each of them holding $\ell=\phi(m) / d$ of the coefficients of $Z$ in its $\ell$ plaintext slots. (Recall that we chose $m=\tilde{O}(\lambda)$ such that $d=O(\log m)$.) Applying the procedure from Figure 1 to these ciphertexts will implicitly apply the bit extraction of Lemma 2 to each plaintext slot, thus leaving us with a collection of $d$ ciphertexts, each holding $\ell$ of the coefficients of $a$ in its plaintext slots. 


\subsection{Step Four: Switching Back to Coefficient Representation}

To finally complete the recryption process, we need to convert the $d$ ciphertexts holding the coefficients of $a$ in their plaintext slots into a single ciphertext that encrypts the polynomial $a$ itself. For this transformation, we appeal to the result of Gentry et al. [11], which says that every depth- $L$ circuit of average-width $\tilde{\Omega}(\lambda)$ and size $T$ can be evaluated homomorphically in time $O(T) \cdot \operatorname{poly}(L, \log \lambda)$, provided that the inputs and outputs are presented in a packed form. Below we show that the transformation we seek can be computed on cleartext by a circuit of size $T=\tilde{O}(m)$ and depth $L=$ polylog $(m)$, and hence (since $m=\tilde{\Theta}(\lambda)$ ) it can be evaluated homomorphically in time $\tilde{O}(m)=\tilde{O}(\lambda)$.

To use the result of Gentry et al. we must first reconcile an apparent "type mismatch": that result requires that both input and output be presented in a packed CRT form, whereas we have input in CRT form but output in coefficient form. We therefore must interpret the output as "something in CRT representation" before we can use the result from [11]. The solution is obvious: since we want the output to be $a$ in coefficient representation, then it is a polynomial that holds the value $A_{j}=a \bmod F_{j}$ in the $j$ 'th slot for all $j$.

Hence the transformation that we wish to compute takes as input the coefficients of the polynomials $a(X)$, and produces as output the polynomials $A_{j}=a \bmod F_{j}$ for $j=0,1, \ldots, \ell-1$. It is important to note that our output consists of $\ell$ values, each of them a degree- $d$ binary polynomial. Since this output is produced by an arithmetic circuit, then we need a circuit that operates on degree- $d$ binary polynomials, in other words an arithmetic circuit over $\mathbb{G F}\left(2^{d}\right)$. This circuit has $\ell \cdot d$ inputs (all of which happen to be elements of the base field $\mathbb{F}_{2}$ ), and $\ell$ outputs that belong to the extension field $\mathbb{G} \mathbb{F}\left(2^{d}\right)$.

Theorem 1. Fix $m \in \mathbb{Z}$, let $d \in \mathbb{Z}$ be the smallest such that $m \mid 2^{d}-1$, denote $\ell=\phi(m) / d$ and let $G \in \mathbb{F}_{2}[X]$ be a degree-d irreducible polynomial over $\mathbb{F}_{2}$ (that fixes a particular representation of $\mathbb{G F}\left(2^{d}\right)$ ). Let $F_{0}(X), F_{1}(X), \ldots, F_{\ell-1}(X)$ be the irreducible (degree-d) factors of the $m$-th cyclotomic polynomial $\Phi_{m}(X)$ modulo 2.

Then there is an arithmetic circuit $\Pi_{m}$ over $\mathbb{F}_{2}[X] / G(X)=\mathbb{G F}\left(2^{d}\right)$ with $\phi(m)$ inputs $a_{0}, a_{1}, \ldots, a_{\phi(m)-1}$ and $\ell$ outputs $z_{0}, z_{1}, \ldots, z_{\ell-1}$, for which the following conditions hold:

- When the inputs are from the base field $\left(a_{i} \in \mathbb{F}_{2} \forall i\right)$ and we denote $a(X)=$ $\sum_{i} a_{i} X^{i} \in \mathbb{F}_{2}[X]$, then the outputs satisfy $z_{j}=a(X) \bmod \left(F_{j}(X), 2\right) \in$ $\mathbb{F}_{2}[X] / G(X)$.

- $\Pi_{m}$ has depth $O(\log m)$ and size $O(m \log m)$.

The proof is in the full version. An immediate corollary of Theorem 1 and the Gentry et al. result [11, Thm. 3], we have:

Corollary 2. There is an efficient procedure that given $d$ ciphertexts, encrypting $d$ polynomials that hold the coefficients of a in their slots, computes a single ciphertext encrypting $a$. The procedure works in time $O(m) \cdot p o l y l o g(m)$ (and uses at most polylog $(m)$ levels of homomorphic evaluation). 


\subsection{Details of Step Two}

The transformation of Step Two is roughly the inverse of the transformation that we described above for Step Four, with some added complications. In this step, we have the polynomial $Z(X)$ over the ring $\mathbb{Z} / 2^{r+1} \mathbb{Z}$, and we view it as defining $\ell$ plaintext slots with the $j$ 'th slot containing $B_{j} \stackrel{\text { def }}{=} Z \bmod \left(F_{j}, 2^{r+1}\right)$. Note that the $B_{j}$ 's are degree- $d$ polynomials, and we consider them as elements in the "extension ring" $R_{2^{r+1}}^{d} \stackrel{\text { def }}{=} \mathbb{Z}[X] /\left(G(X), 2^{r+1}\right.$ ) (where $G$ is some fixed irreducible degree- $d$ polynomial modulo $2^{r+1}$ ).

Analogous to Theorem 1 we would like to argue that there is an arithmetic circuit over $R_{2^{r+1}}^{d}$ that get as input the $B_{j}$ 's (as elements of $R_{2^{r+1}}^{d}$ ), and outputs all the coefficients of $Z$ (which are elements of the base ring $\mathbb{Z} / 2^{r+1} \mathbb{Z}$ ). Then we could apply again to the result of Gentry et al. [11] to conclude that this circuit can be evaluated homomorphically with only polylog overhead.

For the current step, however, the arithmetic circuit would contain not only addition and multiplication gates, but also Frobenius map gates. Namely, gates $\rho_{k}(\cdot)$ (for $k \in$ $\{1,2, \ldots, d-1\})$ computing the functions

$$
\rho_{k}(u(X))=u\left(X^{2^{k}}\right) \bmod \left(G(X), 2^{r+1}\right) .
$$

It was shown in [11] that arithmetic circuits with Frobenius map gates can also be evaluated homomorphically with only polylog overhead. The Frobenius operations being simply an additional automorphism operation which can be applied homomorphically to ciphertexts.

Theorem 2. Fix $m, r \in \mathbb{Z}$, let $d \in \mathbb{Z}$ be the smallest such that $m \mid 2^{d}-1$, denote $\ell=\phi(m) / d$ and let $G(X)$ be a degree-d irreducible polynomial over $\mathbb{Z} / 2^{r+1} \mathbb{Z}$ (that fixes a particular representation of $\left.R_{2^{r+1}}^{d}\right)$. Let $F_{0}(X), F_{1}(X), \ldots, F_{\ell-1}(X)$ be the irreducible (degree- $d$ ) factors of the $m$-th cyclotomic polynomial $\Phi_{m}(X)$ modulo $2^{r+1}$.

Then there is an arithmetic circuit $\Psi_{m, r}$ with Frobenius-map gates over $R_{2^{r+1}}^{d}$ that has $\ell$ input $B_{0}, B_{1}, \ldots, B_{\ell-1}$ and $\phi(m)$ outputs $Z_{0}, Z_{1}, \ldots, Z_{\phi(m)-1}$, for which the following conditions hold:

- On any inputs $B_{0}, \ldots, B_{\ell-1} \in R_{2^{r+1}}^{d}$, the outputs of $\Psi_{m, r}$ are all in the base ring, $Z_{i} \in \mathbb{Z} / 2^{r+1} \mathbb{Z} \forall i$. Moreover, denoting $Z(X)=\sum_{i} Z_{i} X^{i}$, it holds that $Z(X) \bmod$ $\left(F_{j}(X), 2^{r+1}\right)=B_{j}$ for all $j$.

- $\Pi_{m}$ has depth $O(\log m+d)$ and size $O(m(d+\log m))$.

The proof is in the full version. As before, a corollary of Theorem 2 and the result from [11], is the following:

Corollary 3. There is an efficient procedure that given a single ciphertext encrypting $Z^{\prime}$ outputs $d$ ciphertexts encrypting d polynomials that hold the coefficients of $Z^{\prime}$ in their plaintext slots. The procedure works in time $\tilde{O}(m)$ (and uses at most polylog $(m)$ levels of homomorphic evaluation). 


\subsection{An Alternative Variant}

The procedure from Section 4.7 works in time $\tilde{O}(m)$, but it is still quite expensive. One alternative is to put in the public key not just one ciphertext encrypting the $q_{L}$-secret-key $\mathfrak{s}$, but rather $d$ ciphertexts encrypting polynomials that hold the coefficients of $\mathfrak{s}$ in their plaintext slots. Then, rather than using the simple formula from Equation (2) above, we evaluate homomorphically the inner product of $\mathbf{s}=(1, \mathfrak{s})$ and $\mathbf{c}=\left(c_{0}, c_{1}\right)$ modulo $\Phi_{m}(X)$ and $2^{r+1}$. This procedure will be even faster if instead of the coefficients of $\mathfrak{s}$ we encrypt their transformed image under length- $m$ DFT. Then we can compute the DFT of $c_{1}$ (in the clear), multiply it homomorphically by the encrypted transformed $\mathfrak{s}$ (in SIMD fashion) and then homomorphically compute the inverse-DFT and the reduction modulo $\Phi_{m}$. Unfortunately this procedure still requires that we compute the reduction mod- $\Phi_{m}(X)$ homomorphically, which is likely to be the most complicated part of bootstrapping. Finding a method that does not require this homomorphic polynomial modular reduction is an interesting open problem.

Acknowledgments. The first and second authors are sponsored by DARPA and ONR under agreement number N00014-11C-0390. The U.S. Government is authorized to reproduce and distribute reprints for Governmental purposes notwithstanding any copyright notation thereon. The views and conclusions contained herein are those of the authors and should not be interpreted as necessarily representing the official policies or endorsements, either expressed or implied, of DARPA, or the U.S. Government. Distribution Statement "A" (Approved for Public Release, Distribution Unlimited).

The third author is sponsored by DARPA and AFRL under agreement number FA8750-11-2-0079. The same disclaimers as above apply. He is also supported by the European Commission through the ICT Programme under Contract ICT-2007-216676 ECRYPT II and via an ERC Advanced Grant ERC-2010-AdG-267188-CRIPTO, by EPSRC via grant COED-EP/I03126X, and by a Royal Society Wolfson Merit Award. The views and conclusions contained herein are those of the authors and should not be interpreted as necessarily representing the official policies or endorsements, either expressed or implied, of the European Commission or EPSRC.

\section{References}

1. Brakerski, Z., Gentry, C., Vaikuntanathan, V.: Fully homomorphic encryption without bootstrapping. In: Innovations in Theoretical Computer Science, ITCS 2012 (2012), http: / / eprint.iacr.org/2011/277

2. Brakerski, Z., Vaikuntanathan, V.: Efficient fully homomorphic encryption from (standard) LWE. In: FOCS 2011. IEEE Computer Society (2011)

3. Brakerski, Z., Vaikuntanathan, V.: Fully Homomorphic Encryption from Ring-LWE and Security for Key Dependent Messages. In: Rogaway, P. (ed.) CRYPTO 2011. LNCS, vol. 6841, pp. 505-524. Springer, Heidelberg (2011)

4. Cassels, J.W.S.: Local Fields. LMS Student Texts, vol. 3. Cambridge University Press (1986)

5. van Dijk, M., Gentry, C., Halevi, S., Vaikuntanathan, V.: Fully Homomorphic Encryption over the Integers. In: Gilbert, H. (ed.) EUROCRYPT 2010. LNCS, vol. 6110, pp. 24-43. Springer, Heidelberg (2010), Full version available on-line from http://eprint.iacr.org/2009/616 
6. Gentry, C.: A fully homomorphic encryption scheme. $\mathrm{PhD}$ thesis, Stanford University (2009), http: / / crypto.stanford.edu/craig

7. Gentry, C.: Fully homomorphic encryption using ideal lattices. In: Mitzenmacher, M. (ed.) STOC, pp. 169-178. ACM (2009)

8. Gentry, C., Halevi, S.: Fully homomorphic encryption without squashing using depth-3 arithmetic circuits. In: FOCS 2011. IEEE Computer Society (2011)

9. Gentry, C., Halevi, S.: Implementing Gentry's Fully-Homomorphic Encryption Scheme. In: Paterson, K.G. (ed.) EUROCRYPT 2011. LNCS, vol. 6632, pp. 129-148. Springer, Heidelberg (2011)

10. Gentry, C., Halevi, S., Smart, N.P.: Better bootstrapping for fully homomorphic encryption (2011), http://eprint.iacr.org/2011/680

11. Gentry, C., Halevi, S., Smart, N.P.: Fully Homomorphic Encryption with Polylog Overhead. In: Pointcheval, D., Johansson, T. (eds.) EUROCRYPT 2012. LNCS, vol. 7237, pp. 465-482. Springer, Heidelberg (2012), Full version at http: / / eprint.iacr.org/2011/566

12. Rivest, R., Adleman, L., Dertouzos, M.L.: On data banks and privacy homomorphisms. In: Foundations of Secure Computation, pp. 169-180 (1978)

13. Smart, N.P., Vercauteren, F.: Fully Homomorphic Encryption with Relatively Small Key and Ciphertext Sizes. In: Nguyen, P.Q., Pointcheval, D. (eds.) PKC 2010. LNCS, vol. 6056, pp. 420-443. Springer, Heidelberg (2010)

14. Smart, N.P., Vercauteren, F.: Fully homomorphic SIMD operations (2011) (manuscript), http://eprint.iacr.org/2011/133 